\title{
Very Late Stent Thrombosis Caused by Rupture of Lipid-Laden Neointima in a Self-Expanding Coronary Stent
}

\author{
Seiji Koga, ${ }^{1}$ MD, Satoshi Ikeda, ${ }^{1}$ MD, Takeo Yoshida, ${ }^{1}$ MD, Tomoo NaKata, ${ }^{1}$ MD, \\ Masayoshi Takeno, ${ }^{1} \mathrm{MD}$, Yuji KoIde, ${ }^{1} \mathrm{MD}$, and Koji MaEmura, ${ }^{1} \mathrm{MD}$
}

\begin{abstract}
SUMMARY
A 60-year-old man was admitted to our hospital due to acute ST-segment elevation myocardial infarction. He had a history of self-expanding stent implantation in the proximal left anterior descending artery due to stable angina pectoris 7 years earlier. Emergent coronary angiography on admission showed occlusion in the distal portion of the previously stented segment, in which observation by optical coherence tomography revealed the existence of a remarkable proliferation of lipid-laden neointimal tissues with rupture and thrombus. This suggests that very late stent thrombosis in a selfexpanding stent may occur through the process of atheromatous formation. (Int Heart J 2012; 53: 202-204)
\end{abstract}

Key words: Optical coherence tomography, Acute myocardial infarction, Percutaneous coronary intervention

$\mathrm{V}$ ery late stent thrombosis (VLST) has been recognized as a rare, but potentially life-threatening complication after drug-eluting stent (DES) implantation. Recently, VLST after bare-metal stent (BMS) implantation that was different to that observed after DES implantation has also been reported. ${ }^{1)}$ VLST after self-expanding stent implantation has not been reported to the best of our knowledge. Herein we report a case of acute myocardial infarction due to VLST which occurred at 7 years after implantation of a self-expanding stent, evaluated by optical coherence tomography (OCT).

\section{Case Report}

A 60-year-old man was admitted to our hospital with acute ST-segment elevation myocardial infarction. He had undergone implantation with a self-expanding BMS (Radius $4.0 \times 31 \mathrm{~mm}$, Boston Scientific, Natick, MA, USA) in the proximal left anterior descending artery due to stable angina pectoris 7 years previously at another hospital. He was discharged on aspirin (100 mg once daily) and ticlopidine (100 mg twice daily). Follow-up coronary angiography (CAG) at 12 months after the procedure confirmed the absence of in-stent restenosis. Thereafter, he was maintained on antiplatelet therapy with aspirin (100 mg once daily). Follow-up coronary computed tomography (CT) at 6 months before the current admission revealed moderate local proliferation of neointima with low CT attenuation at the distal portion of the stent (Figure 1), but he had no clinical symptoms. His coronary risk factors were current smoking and hypertension which had been controlled well with amlodipine $5 \mathrm{mg}$ once daily.

Emergency CAG upon admission revealed thrombotic occlusion in the distal portion of the previously stented seg- ment (Figure 2A). The occlusion site was easily crossed using a floppy guidewire (Runthrough NS; Terumo, Tokyo). Thrombolysis in myocardial infarction (TIMI) flow grade 2 was obtained by thrombus aspiration using Export XT catheter (Medtronic, Minneapolis, MN, USA), but 99\% stenosis with haziness persisted in the distal portion of the previously stented segment (Figure 2B). Angiographically, the vessel size was significantly larger in the proximal than distal site of the stented segment.

Subsequently, OCT was performed to evaluate this lesion by an occlusion method. It revealed remarkable proliferation of lipid-laden neointimal tissues, which was observed as a diffusely bordered, signal-poor region with overlying a signalrich band, in the distal portion of the stent (Figures 2D-2F). Ruptured lipid-laden neointima and a massive thrombus protruding into the lumen were also identified (Figures $2 \mathrm{E}$ and $2 \mathrm{~F})$. The proximal to mid portion of the stent contained less neointimal tissue (Figure 2C). Stent malapposition and uncovered stent struts were not identified. Stent area measured by OCT was significantly smaller in the distal $\left(13.6 \mathrm{~mm}^{2}\right)$ than in the proximal portion $\left(19.3 \mathrm{~mm}^{2}\right)$.

The lesion was treated by implantation of a further BMS (Liberte $3.0 \times 28 \mathrm{~mm}$; Boston Scientific, Natick, MA, USA) overlapped with the distal portion of the previously implanted stent. The overlapped site was redilated with a $4.0 \times 15 \mathrm{~mm}$ noncompliant balloon at $18 \mathrm{~atm}$, achieving TIMI flow grade 3 . Thereafter, the patient was maintained on $100 \mathrm{mg}$ aspirin once daily and $75 \mathrm{mg}$ clopidogrel once daily, and a statin $(10 \mathrm{mg}$ atorvastatin once daily). He remains symptom-free at 12 months.

From the ${ }^{1}$ Department of Cardiovascular Medicine, Nagasaki University Graduate School of Biomedical Sciences, Nagasaki, Japan.

Address for correspondence: Satoshi Ikeda, MD, Department of Cardiovascular Medicine, Nagasaki University Graduate School of Biomedical Sciences, 1-7-1 Sakamoto, Nagasaki, Nagasaki 852-8501, Japan.

Received for publication January 4, 2012.

Revised and accepted March 16, 2012. 


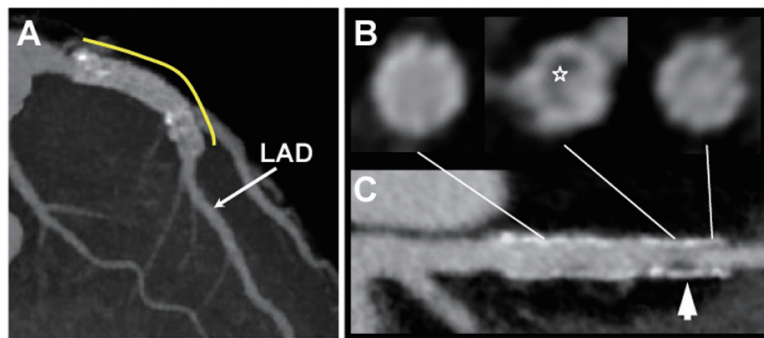

Figure 1. Coronary CT angiography. A: Previously implanted self-expanding stent is located in the proximal LAD (yellow line). B, C: Crosssectional and stretched curved planar reconstructed CT images of stented segment. Focal neointimal proliferation with low CT attenuation (star and white arrowhead) is evident at distal portion of stent. CT indicates computed tomography and LAD, left anterior descending artery.

\section{DiscusSION}

We have presented a case of VLST at 7 years after selfexpanding BMS implantation that developed to acute myocardial infarction. CAG showed acute occlusion in the previously stented segment where the rupture of lipid-laden neointima and thrombosis was observed by OCT.

According to the Academic Research Consortium definition, we diagnosed the present case as definite VLST, which requires the presence of an acute coronary syndrome with angiographic or autopsy evidence of thrombus or occlusion, and onset from > 360 days after stent implantation. ${ }^{2)}$ VLST is related to a high incidence of acute myocardial infarction and mortality. ${ }^{3)}$ Although more common after DES implantation, recent evidence suggests VLST also occurs after BMS implantation. ${ }^{4}$ It is generally thought that lack of stent strut coverage and late malapposition may be responsible for VLST after DES implantation. However, the mechanism of this late catastrophic complication, especially for BMS, is not fully understood.

Neointimal proliferation within BMS generally reaches its peak around 6 months after implantation. Once the stent is covered with neointima, the stented target lesion remains clinically stable. However, recent studies have suggested that the formation and progression of a neoatheroma could occur over a previously healed intrastent. Hasegawa, et al conducted a histopathological analysis using in-stent restenotic tissue retrieved by directional coronary atherectomy, and reported atheromatous degeneration of the neointima followed by plaque rupture occurring up to 5 years after BMS implantation. ${ }^{1)}$ Nakazawa, et al in an autopsy analysis reported that neoatherosclerosis and unstable lesions characterized as thin-cap fibroatheromas or plaque rupture inside BMS were observed after 6 years following implantation. ${ }^{5)}$ Furthermore, recent OCT studies also showed that atherosclerotic changes and consequent plaque vulnerability occurred in the previously stented segments, and its rupture could cause acute coronary syndrome. $^{6-8)}$ Their findings suggest that the formation and progression of a neoatheroma over a previously healed intrastent intima may be one possible mechanism for VLST. In the present case, OCT revealed that rupture of lipid-laden neointima that had formed within 7 years after stent implantation caused stent thrombosis. Of note, coronary CT at 6 months before the current admission revealed moderate local proliferation of neointima at the distal portion of the stent. Neointima

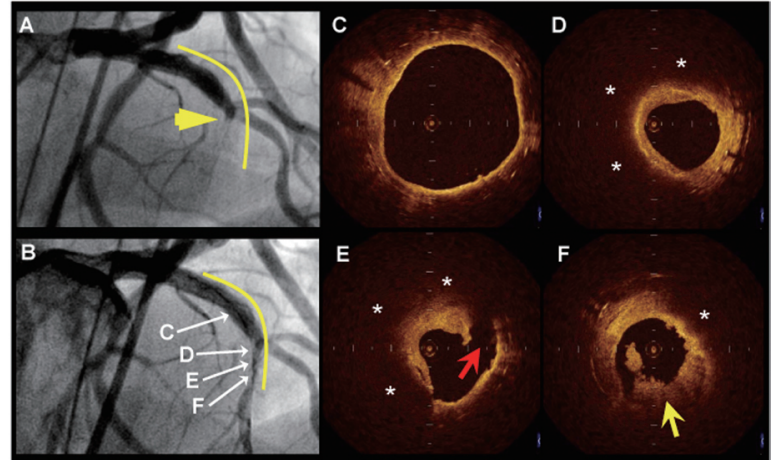

Figure 2. CAG images before (A) and after $(\mathbf{B})$ thrombus aspiration at current admission. Yellow line indicates stented segment. LAD was occluded in distal portion of stent (yellow arrowhead). (C-F) OCT images in the stented segment. Remarkable proliferation of lipid-laden neointima (asterisk), ruptured neointima (red arrow), and thrombus (yellow arrow) were found in distal portion of stent. Less neointima is evident in proximal portion of stent (C). CAG indicates coronary angiography; OCT, optical coherence tomography; and LAD, left anterior descending artery.

inside the stent can become a substrate for an atherogenic process, which may lead to a very late cardiovascular event even a long time after implantation.

A notable finding in the present patient was the localization of atherosclerotic and vulnerable changes in neointima at the distal portion of the self-expanding BMS. Self-expanding stents have the potential advantages of less barotrauma to the vessel wall because of a reduced need for high pressure dilation, differential expansion (to conform to tortuous, tapered or distended segments of the vessel wall), and increased flexibility. ${ }^{9)}$ The self-expanding nature of the Radius stent, together with the long-term shape memory of the nitinol alloy, maintains an outside radial force on the vessel wall until it reaches its final diameter. The Radius stent might have originally been selected for this patient because of the large difference in the diameter of the left anterior descending artery between the proximal and distal sites of the target lesion. However, expansion of a self-expanding stent can dent the media and the vessel becomes progressively damaged. ${ }^{10)}$ Such chronic and continuous deep vessel wall injury may increase subsequent neointimal proliferation. ${ }^{11)}$ At the tapered vessel as in the present patient, chronic damage to the vessel wall from a self-expanding stent might be more severe at the distal site than at the proximal site, because the extent to which a self-expanding stent expands is dependent on the proximal reference diameter. Such differential chronic damage might explain the focal atherosclerotic and vulnerable changes in the neointima at the distal portion of the stent in our patient. In addition, some animal studies indicated that gradual stent expansion may prolong neointimal proliferation to a variable degree even beyond the earlier peak at one to three months in the balloon-expandable stent. ${ }^{12)}$ This prolonged neointimal proliferation may be associated with the very late event at 7 years after stent implantation in the present case.

Strict control of coronary risk factors is also necessary to prevent the formation of neoatheroma inside the stent. In this case, we instructed the patient to cease smoking and introduced statin therapy due to the low-density lipoprotein cholesterol level of $116 \mathrm{mg} / \mathrm{dL}$. Even if the patient remains clinically 
stable, long-term and careful systemic medical therapy will be needed.

\section{REFERENCES}

1. Hasegawa K, Tamai H, Kyo E, et al. Histopathological findings of new in-stent lesions developed beyond five years. Catheter Cardiovasc Interv 2006; 68: 554-8.

2. Mauri L, Hsieh WH, Massaro JM, Ho KK, D'Agostino R, Cutlip DE. N Engl J Med 2007; 356: 1020-9.

3. Ong AT, McFadden EP, Regar E, de Jaegere PP, van Domburg RT, Serruys PW. Late angiographic stent thrombosis (LAST) events with drug-eluting stents. J Am Coll Cardiol 2005; 45: 2088-92.

4. Lemesle G, Pinto Slottow TL, Waksman R. Very late stent thrombosis after bare-metal stent implantation: case reports and review of the literature. J Invasive Cardiol 2009; 21: E27-32.

5. Nakazawa G, Otsuka F, Nakano M, et al. The pathology of neoatherosclerosis in human coronary implants bare-metal and drugeluting stents. J Am Coll Cardiol 2011; 57: 1314-22.

6. Kashiwagi M, Kitabata H, Tanaka A, et al. Very late clinical cardiac event after BMS implantation: in vivo optical coherence tomography examination. JACC Cardiovasc Imaging 2010; 3: 525-7.

7. Qian J, Yang YJ. Very late thrombosis in a bare metal stent: mech- anistic analysis from optical coherence tomography. Catheter Cardiovasc Interv 2012; 79: 288-90.

8. Takano M, Yamamoto M, Inami S, et al. Appearance of lipid-laden intima and neovascularization after implantation of bare-metal stents extended late-phase observation by intracoronary optical coherence tomography. J Am Coll Cardiol 2009; 55: 26-32.

9. Kandzari DE, Goldberg S, Schwartz RS, Chazin-Caldie M, Sketch MH Jr. Clinical and angiographic efficacy of a self-expanding nitinol stent in saphenous vein graft atherosclerotic disease: the Stent Comparative Restenosis (SCORES) Saphenous Vein Graft Registry. Am Heart J 2003; 145: 868-74.

10. Yu ZX, Tamai H, Kyo E, et al. Comparison of the self-expanding Radius stent and the balloon-expandable Multilink stent for elective treatment of coronary stenoses: a serial analysis by intravascular ultrasound. Catheter Cardiovasc Interv 2002; 56: 40-5.

11. Kobayashi Y, Honda Y, Christie GL, et al. Long-term vessel response to a self-expanding coronary stent: a serial volumetric intravascular ultrasound analysis from the ASSURE Trial. A Stent vs. Stent Ultrasound Remodeling Evaluation. J Am Coll Cardiol 2001; 37: 1329-34

12. Hong MK, Beyar R, Kornowski R, Tio FO, Bramwell O, Leon MB. Acute and chronic effects of self-expanding nitinol stents in porcine coronary arteries. Coron Artery Dis 1997; 8: 45-8. 